\title{
Trasplante cardíaco y su relación con el cáncer de piel de tipo melanoma y no melanoma
}

Heart transplantation and its relationship to the type melanoma skin cancer and melanoma

\author{
Claudia Marcela Gaviria ${ }^{1}$, Ana Paula Giraldo ${ }^{1}$, Sol Beatriz Jiménez ${ }^{2}$ \\ 1. Médica; residente de Dermatología, Universidad CES, Medellín, Colombia \\ 2. Médica dermatóloga; jefe, Servicio de Dermatología, Universidad CES, Medellín, Colombia
}

\section{Resumen}

El trasplante cardíaco es una cirugía que cada vez se hace con mayor frecuencia en nuestro medio. En la Clínica Cardio VID de Medellín aumenta cada año el número de pacientes a quienes se les practica este procedimiento. Ellos son candidatos al desarrollo de tumores de piel por su estado de inmunosupresión crónica, el cual aumenta si existen condiciones previas. La mayoría son pacientes mayores de 50 años de edad que han vivido en el trópico con poca fotoprotección y está demostrado que el carcinoma escamocelular es más frecuente en ellos.

La intervención dermatológica temprana es uno de los factores de mayor impacto que se ha documentado en múltiples estudios y, a pesar de ello y de tener en cuenta que es uno de los principales tipos de cáncer junto con el linfoma, existe poca atención a la piel de estos pacientes.

PALABRAS CLAVE: cáncer de piel, trasplante de corazón, inmunosupresión.

\author{
Correspondencia: \\ Claudia Marcela Gaviria \\ Email: \\ claugavio2@hotmail.com \\ Recibido: 22 de septiembre de 2014 . \\ Aceptado: 20 de octubre de 2014.
}

No se reportan conflictos de interés.

\section{Summary}

Heart transplantation is a surgery that is becoming more frequent in our environment. At the Clínica Cardio VID of Medellin the number of patients undergoing this procedure increases every year, and they become candidates for the development of skin tumors given their state of chronic immune suppression, which increases if there are preconditions. Most patients are older than 50 years of age who have lived in the tropics with little photoprotection and evidence that squamous cell carcinoma is more common in these patients.

The early dermatological intervention is one of the factors with the greatest impact has been documented in multiple studies and despite this and consider it one of the main types of cancer along with lymphoma, is very little intervention in this area.

KEY WORDS: skin cancer, heart transplantation, immunosuppression.

\section{Introducción}

El tema de los trasplantes de órganos es uno de los más importantes en los últimos tiempos. Uno de los avances más significativos de la cirugía moderna, es el trasplante alogénico de órganos vitales ${ }^{1}$.
El trasplante de corazón está indicado en diversos tipos de insuficiencia cardiaca en fase terminal y en lesiones cardiacas irreparables, condiciones generalmente presentes en pacientes con alto riesgo de muerte por falla cardiaca resistente al tratamiento ${ }^{1}$. 


\section{Trasplante cardíaco}

Desde que se practicó el primer trasplante de riñón en el Peter Bent Brigham Hospital de Boston en 1951, se han llevado a cabo con éxito numerosos trasplantes de riñón. También, se ha tenido éxito en trasplantes de dientes, córneas, hígado, corazón y glándulas endocrinas ${ }^{1}$.

Christian Barnard llevó a cabo el primer trasplante cardiaco de humano a humano el 3 de diciembre de 1967 en el Groote Schuur Hospital de Ciudad del Cabo (Sudáfrica). El paciente era Louis Washkansky y el donante fue Denise Darvall, quien se encontraba en muerte cerebral tras un accidente automovilístico. El segundo trasplante se le practicó, el 2 de enero de 1968, a Philip Blaiberg, que vivió 563 días después de la intervención ${ }^{1}$.

En Suramérica, el primer trasplante exitoso lo practicaron el cirujano Jorge Kaplán y su equipo, el 28 de junio de 1968. Sucedió en el antiguo Hospital Naval Almirante Nef de Valparaíso (Chile) y la paciente que recibió el trasplante fue María Elena Peñaloza, una joven de 24 años con una cardiomiopatía dilatada.

En Colombia, el primero se llevó a cabo en 1985 en la Clínica Cardiovascular Santa María de Medellín. En la actualidad, existen siete centros de trasplante cardíaco distribuidos en el territorio nacional, en ciudades como Bogotá, Medellín y Cali. A nivel mundial, España es el país que ocupa el primer lugar en número de trasplantes por mil habitantes².

\section{EPIDEMIOLOGÍA}

Las neoplasias de órgano sólido son una de las principales causas de muerte entre los receptores de trasplante y es consecuencia del uso de inmunosupresores ${ }^{1}$.

La población con trasplantes tiene un riesgo de 10 a 100 veces mayor de desarrollar cáncer de cualquier tipo, que la población general (incidencia general, de 5 a $6 \%$ ). Los tumores más frecuentes son los de piel y los linfomas. El cáncer de piel ocupa un porcentaje importante; es de 42 a $50 \%$ de todas las neoplasias malignas ${ }^{3,4}$. A su vez, los pacientes con trasplante de corazón son dos veces más propensos a desarrollar neoplasias malignas de piel que aquellos con trasplante renal; esto se debe al grado de inmunosupresión en que se encuentran, el cual es mayor por los medicamentos que reciben. Lo anterior se ve reflejado en el tiempo trascurrido entre el trasplante y el diagnóstico del cáncer de piel, ya que es mucho más corto para el receptor de trasplante cardiaco que para aquel con trasplante renal (3,9 años frente a 8,6 años). Se espera, entonces, que en la medida que aumente la expectativa de vida de los pacientes con trasplantes, aumente también la incidencia de tumores ${ }^{5,6}$.

El tiempo promedio de aparición del cáncer después del trasplante, según la edad, es de ocho años para los menores de 40 años y de 3 años para los mayores de 50 años $^{7}$ y, según el tiempo transcurrido desde el trasplante hasta el momento del diagnóstico, es de 9,1 por 1.000 personas al año en los primeros cuatro años, de 12,2 , entre los cinco y los ocho años, y de 20,2, por encima de los ocho años ${ }^{3,8,9}$. En otros estudios se reportan incidencias a 5, 10, 20 y 30 años, de 11, 25, 54 y $74 \%$, respectivamente 9 .

En individuos sanos, el carcinoma basocelular es más común que el carcinoma escamocelular. Sin embargo, la incidencia del carcinoma escamocelular en receptores de trasplante es de 65 a 250 veces mayor que en la población general, mientras que la incidencia de carcinoma basocelular en estos individuos es tan solo 10 veces mayor ${ }^{10-13}$.

Para la población general, la relación entre carcinoma basocelular y carcinoma escamocelular es de 5 a $1 \mathrm{y}$, en la población con trasplantes, es de 1 a 1,8-2,3 (rango: 1:1,2 a 1:15) ${ }^{5,14}$. El carcinoma escamocelular es más prevalente en los sometidos a trasplante e inmunosupresión $y$, en menor grado, lo es el carcinoma escamocelular ${ }^{3,9,15}$. Esto podría ser de importancia, debido a que en la intervención y posible prevención del cáncer sería de mucho más impacto la fotoprotección para el carcinoma escamocelular, sobre todo si se tiene en cuenta que este tipo de cáncer es más agresivo que el basocelular. Por esta razón, la fotoprotección se debe instaurar de una manera efectiva en los receptores de trasplantes ${ }^{16}$.

Respecto al melanoma, se sabe que es la neoplasia maligna de piel menos común en pacientes con trasplante, aunque es claro que el tratamiento inmunosupresor es un factor de riesgo para melanoma maligno y, por esta razón, el melanoma es más frecuente en casos de trasplante que en individuos sanos, aunque el escamocelular es el más frecuente en esta población ${ }^{17}$. El melanoma corresponde al 6,2\% del cáncer de piel en adultos con trasplante y, al $15 \%$, en niños. El intervalo entre el momento del trasplante y su diagnóstico es de cinco años. En pacientes con melanoma antes del trasplante, el riesgo de recurrencia posterior es de $20 \% 0^{7}$.

En el registro científico de trasplantes de Estados Unidos, de 1987 a 2008, se tiene la cohorte más amplia hasta el momento; en ella se encontró que, de 175.732 pacientes, 381 sufrieron melanoma, lo que representa un número 2,6 veces mayor que en la población general ${ }^{18}$.

En los reportes de los registros de la Mayo Clinic, se encontró un rango de 5,5 años entre el trasplante y la aparición del melanoma ${ }^{19}$.

El carcinoma escamocelular es más agresivo y mortal en casos de trasplante que en individuos que no reciben inmunosupresores; de una mortalidad de 5,1\% por cáncer de piel, 60 \% es por cáncer escamocelular 
y 33\% por melanoma. Esto es contrario a lo que sucede en la población general, en la cual el melanoma es la primera causa de muerte 5,9 .

\section{CONDICIONES INMUNOLÓGICAS}

Los principales problemas que se presentan en el trasplante de órganos no proceden de la técnica quirúrgica, sino del rechazo del órgano donado por el sistema inmunológico del receptor y las complicaciones relacionadas con el tratamiento inmunosupresor. El rechazo se presenta por activación de la respuesta alogénica, en la cual los linfocitos $\mathrm{T}$ del receptor se activan al reconocer diferencias en el sistema HLA (Human Leucocyte Antigen) entre el donante y el receptor.

El alotrasplante se reconoce por dos vías. En la vía directa, las células presentadoras de antígeno del órgano trasplantado presentan antígenos a los linfocitos $\mathrm{T}$ del receptor. En la vía indirecta, las células del receptor presentan los antígenos del órgano trasplantado, después de fagocitar células apoptóticas derivadas de dicho órgano. Como se mencionó anteriormente, esta reacción se presenta especialmente por diferencias mayores y menores en el sistema HLA.

Debido a este reconocimiento del trasplante y a la activación del sistema inmunitario, es indispensable el uso de inmunosupresores para evitar el rechazo del órgano trasplantado. En el caso de órganos sólidos, como corazón y riñón, el tratamiento inmunosupresor se requiere de forma permanente, dado que a la fecha no ha sido posible establecer protocolos que induzcan tolerancia especifica al órgano trasplantado que permitan retirar los inmunosupresores de forma segura para la funcionalidad del órgano. Aun con el uso de inmunosupresores, pueden presentarse episodios de rechazo que se evidencian por las biopsias o la disfunción del órgano.

Los riesgos del tratamiento inmunosupresor son dos, especialmente, el riesgo de infecciones y de desarrollo de neoplasias después del trasplante, muchas de ellas asociadas a infecciones virales ${ }^{20,21}$.

\section{BIOPSIA ENDOMIOCÁRDICA}

La biopsia endomiocárdica es una técnica diagnóstica percutánea, que consiste en extraer pequeñas muestras del músculo cardiaco por medio de un biótomo. Este procedimiento es fundamental para la detección del rechazo humoral o celular del injerto, ya que los síntomas y signos clínicos, y los métodos diagnósticos no invasivos disponibles, son poco específicos. Este procedimiento se considera seguro y exitoso en más del $90 \%$ de los casos. La incidencia de complicaciones varía de $1 \mathrm{a}$ $6 \%$, con mortalidad de $0,4 \%$. El resultado de la biopsia endomiocárdica permite descartar o diagnosticar la presencia de rechazo humoral o celular del injerto. De igual forma, permite evaluar el resultado del tratamiento instaurado, según el tipo de rechazo y su gravedad ${ }^{1}$.

La biopsia endomiocárdica obtenida por vía percutánea fue descrita por primera vez por Sakakibara en 1962. En 1984, la Organización Mundial de la Salud (OMS) reconoció a la biopsia endomiocárdica como un método que permite confirmar el diagnóstico clínico sospechado, al permitir hallazgos anatomopatológicos que llegan a ser de gran utilidad ${ }^{1}$.

\section{Cáncer}

El cáncer es una enfermedad en la cual un grupo de células de determinado sistema inician una multiplicación descontrolada y acelerada, debido a la alteración en la función de los genes y proteínas reparadoras del ADN, lo cual imposibilita la corrección de los defectos celulares y lleva a alteración de la función celular, muerte celular y formación tumoral no controlada, llegando a invadir otros tejidos de los cuales no forman parte. Además de esto, pueden llegar a sitios distantes por medio de los vasos sanguíneos y el sistema linfático ${ }^{22-24}$.

Tan sólo en el 2007, 7,6 millones de personas murieron de cáncer en todo el mundo. Según datos estadísticos del Ministerio de Salud y Protección Social de Colombia, cada año se presentan 70.877 casos nuevos de la enfermedad, con una incidencia de 186,6 por 100.000 hombres y 196,9 por 100.000 mujeres; los más frecuentes son el de próstata en hombres y el de mama en mujeres. Se estima que para el 2030, cada año se presentarán 124.000 nuevos casos, lo cual platea un panorama de retos y obstáculos por resolver.

Para el resto del mundo no es diferente; el cáncer ha pasado a ser un importante problema de salud pública en los países en desarrollo, igualando así su efecto en la mortalidad y morbilidad de los países industrializados ${ }^{25}$. En el 2000, de los 56 millones de fallecimientos por todas las causas, el cáncer fue responsable del $12 \%$ y lo más importante es que los estudios demuestran que esta incidencia aumentará hasta en $50 \%$ para el año 2020, en el que se presentarían 15 millones de nuevos casos ${ }^{25}$.

\section{CÁNCER DE PIEL}

El de piel es uno de los tipos de cáncer más comunes alrededor del mundo. Su frecuencia varía según la región geográfica, el fototipo de piel, la edad (mayores de 40 a 45 años), la exposición solar (principalmente, rayos ultravioleta B), la exposición a cámaras de bronceo, la predisposición y el sexo masculino, entre otros factores ${ }^{3,14}$. La incidencia del cáncer de piel está aumentando a gran 


\section{"En los pacientes que reciben trasplante de un órgano sólido, se ha documentado mayor frecuencia de cáncer de piel que en la población general".}

escala, principalmente el no melanoma. Corresponde a una tercera parte de todos los cánceres en el mundo y es el primero en Estados Unidos con un estimativo de un millón de casos nuevos cada año, pero, aunque esta incidencia haya aumentado, durante los últimos años se ha logrado un gran impacto en la mortalidad ${ }^{26}$.

El tipo más común de cáncer de piel es el no melanoma, es decir, los carcinomas basocelular y escamocelular que, por fortuna, son los tumores de piel más fáciles de diagnosticar y tratar. Estos se desarrollan en la región germinativa de la epidermis, en los queratinocitos o las estructuras anexiales (folículos pilosos o glándulas sudoríparas) ${ }^{26}$.

\section{CÁNCER de PIEL EN PACIENTES RECEPTORES DE TRASPLANTE}

En los pacientes que reciben trasplante de un órgano sólido, se ha documentado mayor frecuencia de cáncer de piel que en la población general. Este fenómeno se debe a múltiples factores que confluyen, formando un ambiente en el que las células se vuelven más propensas a la mutagénesis.

Entre los principales factores de riesgo, que se discutirán más adelante, se encuentran: la inmunosupresión inducida después del trasplante (azatioprina, ciclosporina, etc.), la edad de los pacientes (generalmente, mayores de 50 años al momento del trasplante), el sexo (mayor incidencia en hombres) y la falta de protección frente a la radiación ultravioleta ${ }^{9,27,28}$.

Probablemente, el aumento en la incidencia de cáncer de piel en este tipo de pacientes se deba a una mayor supervivencia después del trasplante ${ }^{29}$.

Este mayor riesgo de cáncer de piel conlleva gran cantidad de implicaciones, ya que, además de representar un aumento en la mortalidad, genera grandes repercusiones en la calidad de vida de quien lo padece, pues causa dolor sustancial, desfiguración, pérdida o disminución de la función y el rango de movilidad, incapacidad laboral, metástasis e, incluso, la muerte del paciente ${ }^{3,8,23}$.

El cáncer de piel es la neoplasia maligna más común (42 a $50 \%$ ) en los receptores de trasplante cardiaco, en el hemisferio norte ${ }^{3,8,30,31} \mathrm{Su}$ incidencia aumenta después del trasplante: es de 9,1 por 1.000 personas al año en los primeros cuatro años y se incrementa a 12,2 entre los cinco y los ocho, y a 20,2 después de los ocho años ${ }^{3}$.

Como se mencionó previamente, es importante saber que la relación entre el carcinoma basocelular y el escamocelular se invierte en los pacientes con trasplante; el escamocelular se vuelve más frecuente, y la proporción entre basocelular y escamocelular aumenta de 5:1 a 1:2; además el escamocelular es más agresivo que en la población general $5,9,14$.

\section{FACTORES PREDISPONENTES PARA CÁNCER DE PIEL EN RECEPTORES DE TRASPLANTE CARDÍACO}

\section{Tratamiento inmunosupresor}

Algunos inmunosupresores usados después de trasplantes, se han implicado fuertemente en mutaciones genéticas y daños en la reparación del ADN. La ciclosporina produce inhibición de los genes reparadores del ADN y promueve el crecimiento tumoral por un mecanismo no inmunitario, al estimular la producción de factor de crecimiento transformante beta (TGF- $\beta)^{3,8}$. La azatioprina ha demostrado ser un compuesto sensibilizador a los rayos ultravioleta A y producir daño oxi- 
dativo mutagénico. Además, se ha demostrado que los fármacos usados para la inducción temprana de inmunosupresión para evitar el rechazo inmediato del trasplante, como la globulina antitimocito (ATG) y el anti$\mathrm{CD}_{3}$ (OKT3), pueden tener un papel en la patogenia del cáncer de piel ${ }^{11,16}$. El tacrolimus tiene propiedades inmunosupresoras similares a las de la ciclosporina, pero más potentes, aunque se ha visto una incidencia similar que con los demás inmunosupresores en el desarrollo de cáncer de piel ${ }^{8}$.

Los estudios indican que el riesgo de carcinoma escamocelular en casos de trasplante, es directamente proporcional a la carga inmunosupresora (incluyendo ciclosporina, azatioprina, OKT3 y ATG). Además, demuestran que el tratamiento inmunosupresor a largo plazo conlleva un mayor riesgo de carcinoma escamocelular que a corto plazo ${ }^{10,16}$.

En un estudio italiano del 2004 sobre el grado de inmunosupresión y factores de riesgo para cáncer de piel en receptores de trasplante cardiaco, Belloni, et al., sugieren que el riesgo del carcinoma escamocelular, mas no el del basocelular, se relaciona estrechamente con el grado de inmunosupresión global, más que con un determinado medicamento ${ }^{3,32}$. Además, en varios estudios también se ha relacionado la duración de la inmunosupresión ${ }^{12,33}$.

\section{Lesiones precursoras previas}

En algunos estudios alrededor del mundo, se han relacionado algunas lesiones precursoras previas al trasplante con el posterior desarrollo de cáncer de piel ${ }^{34}$. Las queratosis actínicas son precursoras de las neoplasias de piel (carcinomas escamocelular y basocelular) y son responsables de un riesgo tres veces mayor ${ }^{5,7,11}$.

El papel del virus del papiloma humano (Human papillomavirus, HPV) está demostrado en la carcinogénesis. El carcinoma escamocelular se asocia con este virus e, histológicamente, con características de la infección por HPV. El ADN de este virus se detecta en 65 a $90 \%$ de los carcinomas escamocelulares en receptores de trasplante ${ }^{7,9,16}$.

\section{Exposición a la luz solar}

Está bien establecido que la exposición solar es un factor de riesgo de alto impacto para el desarrollo de cáncer de piel no melanoma en receptores de trasplante, ya que la radiación ultravioleta produce un daño directo en el ADN celular y, a su vez, tiene un efecto inmunosupresor que, sumado a la quimioterapia que debe administrarse, aumentan el riesgo de carcinoma escamocelular 65 veces más que el de la población general y, para el basocelular, 10 veces más ${ }^{35}$.
El carcinoma escamocelular es más prevalente en las áreas de la piel expuestas a la luz solar, como brazos, 'v' del cuello y cuero cabelludo. Por el contrario, el carcinoma basocelular es más frecuente en el tronco y, en esta población, tiene menor relación, aunque no nula, con la exposición a la radiación ultravioleta ${ }^{8}$. Los receptores de trasplante tienen 21 veces más riesgo de cáncer de piel en las áreas expuestas a la luz que la población general, en comparación con áreas no expuestas, en las que el aumento del riesgo es solo cuatro a siete veces mayor ${ }^{35,5,11}$. Se ha evidenciado que el daño solar antes de los 30 años, es un factor de riesgo mayor para desarrollar carcinoma escamocelular después del trasplantes.

También, cabe recalcar que no sólo las áreas expuestas a la luz, sino también el tiempo acumulado de exposición solar, tienen estrecha relación con la aparición de esta enfermedad. Por lo tanto, el hecho de vivir en una zona con alta radiación solar, favorece la aparición más frecuente de cáncer de piel en dicha población ${ }^{3,21}$.

\section{Edad}

La edad al momento del trasplante es un factor muy importante que se debe tener en cuenta a la hora de evaluar la prevalencia del cáncer de piel, ya que se ha evidenciado en múltiples estudios su papel promotor de esta enfermedad $^{30,33}$. En el estudio de Alida, et al., del 2000 de factores de riesgo de cáncer de piel en receptores de trasplante cardiaco, se demostró que la edad mayor de 50 años aumenta 7,3 veces más el riesgo de desarrollar cáncer. También, se demostró que no sólo es el factor de riesgo más importante, sino que también hay una disminución en el intervalo de tiempo entre el momento del trasplante y la presentación de la primera lesión ${ }^{21}$.

\section{Falta de educación}

Por otra parte, en diferentes estudios se ha tratado de evaluar las razones por las que este tipo de cáncer ha aumentado tanto en los receptores de trasplante y se ha demostrado que la falta de un asesoramiento adecuado, el desconocimiento de la importancia de la protección solar y la falta de compromiso de los pacientes y el propio médico frente a este tema, son las principales razones del poco impacto que ha tenido el uso de protector solar en la prevención del cáncer ${ }^{16,36}$.

Se argumenta, entonces, que la educación del paciente sobre los efectos de la radiación ultravioleta y las diferentes formas de evitarla, es una meta importante a la hora de prevenir la aparición de cáncer de piel no melanoma ${ }^{35}$.

Se han llevado a cabo diversos estudios en los que se demuestra que la interacción e instrucción cara a cara 
del paciente son de gran importancia y tienen un gran efecto e impacto en la percepción que este tiene sobre la necesidad de la protección solar ${ }^{37-39}$.

\section{Tratamiento}

El manejo del cáncer de piel, tanto en receptores de trasplante como en individuos sanos, debe iniciarse por la prevención. Al paciente con trasplante se le debe instruir sobre la importancia de la protección solar continua, de manera que sea consciente de los cambios necesarios en su estilo de vida, desde antes de ser sometido al trasplante. Además, una consulta dermatológica inicial y previa a la intervención quirúrgica, es indispensable, ya que de esta manera el médico hará una evaluación inicial y tratará las lesiones precursoras, antes de que la inmunosupresión acelere su evolución hacia una lesión maligna ${ }^{15}$.

El tratamiento de elección en el cáncer de piel es la resección completa del tumor con márgenes de seguridad (respecto al subtipo histológico). En esta población especial, es el mismo que para los pacientes no inmunosuprimidos, es decir, existen diferentes alternativas que se escogen según el tipo de paciente, el tamaño de la lesión, la edad, el tipo histológico del tumor y la localización anatómica por zonas, según correspondan a un área de alto, mediano o bajo riesgo ${ }^{26}$.

Existen dos modalidades terapéuticas: los métodos con control histológico de márgenes, como la cirugía convencional y micrográfica de Mohs, y métodos sin control histológico, como criocirugía, doble electro-curetaje, inmunoterapia y quimioterapia tópicas (5-fluoruracilo, imiquimod), y radioterapia local $4^{\circ}$. El imiquimod debe reservarse para lesiones pequeñas, ya que su aplicación en grandes zonas puede llevar a la activación sistémica de la inmunidad innata, con posibles riesgos de rechazo del trasplante. La terapia fotodinámica es de utilidad en el tratamiento del fotodaño y las lesiones precursoras de tipo queratosis actínicas.

Mención especial requiere el carcinoma escamocelular, por tratarse de un tumor que tiene mayor riesgo de metástasis a distancia; este riesgo se aumenta en los receptores de trasplante, por lo que siempre se debe evaluar el riesgo de recurrencia, o metástasis local o a distancia ${ }^{14}$, para lo cual hay que tener en cuenta ciertos factores (tamaño del tumor, subtipo histológico, espesor del tumor, e invasión neural, linfática o vascular) que influyen en la elección del tratamiento, ya que, de no tenerlos presentes, se podrían presentar recurrencias y metástasis en un porcentaje considerable de los pacientes ${ }^{31}$.

En los pacientes con múltiples verrugas vulgares y desarrollo de carcinomas, se deben considerar cambios en el perfil de los inmunosupresores, usando preferiblemente rapamicina o sirolimus, que tiene un efecto inmunomodulador, en lugar de la ciclosporina. En diversos estudios, este medicamento ha demostrado producir regresión en las lesiones precursoras de cáncer de piel (queratosis actínicas), demostrando una disminución de la incidencia de cáncer de piel de 71,9 \% (en tratamientos convencionales) a 23,8 \% (cuando se cambia a sirolimus) y disminuyendo de esta forma la morbimortalidad asociada ${ }^{41}$.

El uso de retinoides sistémicos en el manejo preventivo del cáncer de piel en receptores de trasplante de órgano sólido, se ha considerado recientemente como una de los tratamientos adyuvantes asociándolo a fotoprotección y evaluación dermatológica periódica ${ }^{42}$. El motivo por el cual se ha empleado este tratamiento es por el efecto de los retinoides en la regulación de la transcripción génica, que llevan finalmente a disminución del crecimiento celular y, por esto, a evitar la transformación maligna. El potencial de este medicamento en la quimioprevención del cáncer de piel en receptores de trasplante de órgano sólido, es promisorio, contribuyendo significativamente a disminuir la morbimortalidad en esta población ${ }^{42}$.

Se han llevado a cabo numerosos estudios en receptores de trasplante de órgano sólido (renal y cardíaco) y con queratosis actínicas múltiples, a quienes se les ha suministrado acitretín, observándose disminución de la evolución a cáncer de piel a los seis meses de iniciado el tratamiento, en comparación con aquellos pacientes a quienes no se les suministró este medicamento. Con esto se concluye que los retinoides sistémicos son una opción en la quimioprevención del cáncer de piel en los pacientes con trasplante, siempre asociados con adecuada protección solar y evaluaciones dermatológicas periódicas ${ }^{42}$.

\section{Conclusiones}

Se justifican los trasplantes por su carácter humanitario, ya que son la posibilidad de prolongar la vida de una persona después de que se ha agotado el manejo médico, además de mejorar la calidad de vida.

Los pacientes con trasplante son una población especial, con significativo mayor riesgo de desarrollar cáncer de piel, que los puede llevar hasta la muerte, especialmente el carcinoma escamocelular.

En los pacientes candidatos a trasplante, se recomienda un examen previo y exhaustivo de la piel, practicado por el dermatólogo, el uso diario de protector solar y el tratamiento agresivo de las lesiones precancerosas. 


\section{Referencias}

1. Beltrán J, Franco C, Echeverría L, Gómez EA, Fernández D, Gómez JE, et al. Guías colombianas de cardiología. Trasplante cardiaco. Rev Colomb Cardiol. 2009;16:29-69.

2. Mendoza F. Aspectos ético-legales del trasplante de corazón. Rev Colomb Cardiol. 2007;14:259-75.

3. Naldi L, Fortina AB, Lovati S, Barba A, Gotti E, Tessari G, et al. Risk of melanoma skin cancer in Italian organ transplant recipients. A registry-based study. Transplantation. 2000;70:1479-84.

4. Krynitz B, Edgren G, Lindelöf B, Baecklund E, Brattström C, Wilczek $\mathrm{H}$, et al. Risk of skin cancer and other malignancies in kidney, liver, heart and lung transplant recipients 1970 to 2008 -a Swedish population-based study. Int J Cancer. 2013;132:1429-38.

5. Wu J, Orengo I. Squamous cell carcinoma in solid-organ trasplantation. Dermatol Online J. 2002;8:4.

6. Ubilla M, Mastrobuoni S, Martín A, Cordero A, Alegría E, Gaviria J, et al. Heart transplant. Ann Sist Sanit Navar. 2006;29:63-78.

7. Euvrard S, Kanitakis J, Claudy A. Skin cancers after organ transplantation. N Engl J Med. 2003;348:1681-91.

8. Chen PL, Chang HH, Chen IM, Lais ST, Shin CC, Weng ZC, et al. Malignancy after heart transplantation. J Chin Med Assoc. 2009;72:588-93.

9. Harwood CA, Mesher D, McGregor JM, Mitchell L, Leedham-Green $\mathrm{M}$, Raftery M, et al. A surveillance model for skin cancer in organ transplant recipients: A 22-year prospective study in an ethnically diverse population. Am J Transpl. 2013;13:119-29.

10. Molina BD, Leiro Mg, Pulpón LA, Mirabet S, Yañez JF, Bonet LA et $a l$. Incidence and risk factors for nonmelanoma skin cancer after heart transplantation. Transpl Proc. 2010;42:3001-5.

11. Geusau A, Dunkler D, Messeritsch E, Sandor N, Heidler G, Rodler $\mathrm{S}$, et al. Non melanoma skin cancer and its risk factors in Austrian population of heart transplant recipients receiving induction therapy. Int J Dermatol. 2008;47:918-25.

12. Liu AS, Eriksson E. Four hundred seventeen squeamous cell cancers in a heart transplant patient. Ann Plast Surg. 2011;67:545-6.

13. Alter M, Satzger I, Schrem H, Kaltenborn A, Kapp A, Gutzmer R. Non-melanoma skin cancer is reduced after switch of immunosuppression to mTOR-inhibitors in organ transplant recipients. I Dtsch Dermatol Ges. 2014;12:480-8.

14. Yagdi T, Sharples L, Tsui S, Large PJ. Malignancy after heart transplantation: Analysis of 24-year experience at a single center. J Card Surg. 2009;24:572-9.

15. Ulrich C, Jurgensen JS, Degen A, Hackethal M, Ulrich M, Patel MJ, et al. Prevention of non-melanoma skin cancer in organ transplant recipients by regular use of a sunscreen: A 24 months, prospective, case-control study. Br J Dermatol. 2009;161:78-84.

16. Tessari G, Girolomoni G. Nonmelanoma skin cancer in solid organ transplant recipients: Update on epidemiology, risk factors and management. Dermatol Surg. 2012;38:1622-30.

17. Ali FR, Lear JT. Melanoma in organ transplant recipients: Incidence, outcomes and management considerations. J Skin Cancer. 2012;2012:1-5.

18. Engels EA, Pfeiffer RM, Fraumeni JR Jr, Kasiske BL, Israni AK, Snyder JJ, et al. Spectrum of cancer risk among US solid organ transplant recipients. JAMA. 2011;306:1891-901.

19. Brewer JD, Christenson LJ, Weaver AL, Dapprich DC, Weenig RH, Lim KK, et al. Malignant melanoma in solid transplant recipients: Collection of database casesand comparison with surveillance, epidemiology and end results data for outcome analysis. Arch Dermatol. 2011;147:790-6.

20. A.K. Abbas, A.K. Lichtman, E.S. Pillai. Inmunología Celular y Molecular. $7^{\text {a }}$ edición, Elsevier Saunders. 2012.

21. Caforio AL, Fortina AB, Piaserico S, Alaibac M, Tona F, Feltrin $\mathrm{G}$, et al. Skin cancer in heart transplant recipients: Risk factor analysis and relevance of immunosuppressive therapy. Circulation. 2000;102(Suppl.3):III222-7.

22. Goldsmith L, Katz S, Gilchrest B, Paller A, Leffell D, Wolff K. Fitzpatrick's Dermatology in General Medicine. 2 vols. 8a ed. United States of America: McGraw Hill Professional; 2012:1239 1259.

23. Harrison TR, Petersdorf RG, Resnick WR, Wilson JD, Wintrobe MM, Martin JB. Harrison's Principles of Internal Medicine. 2vols 16a ed.United States of America: McGraw-Hill; 2005: 501 - 507.

24. Marco Legal: documentos técnicos y estadísticas. Fecha de consulta: 4 de junio de 2014. Disponible en: http://www.ins.gov.co/ lineas-de-accion/Red-Nacional-Laboratorios/Paginas/marcoLegal-documentos-tecnicos-y-estadisticas.aspx

25. Firnhaber JM. Diagnosis and treatment of basal cell and squamous cell carcinoma. Am Fam Physician. 2012;86:161-8.

26. Wagner JA. Cancer after heart transplant: Implications for practice. Prog Transplant. 2012;22:374-8.

27. Rüegg CP, Graf N, Mühleisen B, Szucs TD, French LE, Surber C, et al. Squamous cell carcinoma of the skin induces considerable sustained cost of care in organ transplant recipients. J Am Acad Dermatol. 2012;67:1242-9.

28. Rinaldi M, Pellegrini C, D`Armini AM, Aiello M, Negri M, Arbustini E, et al. Neoplastic disease after heart transplantation: Single center experience. Eur J Cardiothorac Surg. 2001;19:696-701.

29. Alam M, Brown RN, Silber DH, Mullen GM, Feldman DS, Oren $\mathrm{RM}$, et al. Increased incidence and mortality associated with skin cancers after cardiac transplant. Am J Transpl. 2011;11:1488-97.

30. Crespo-Leiro MG, Alonso-Pulpon L, Vásquez JA, Almenar L, Arizón JM, Brossa V, et al. Malignancy after heart transplantation: Incidence, prognosis and risk factors. Am J Transpl. 2008;8:10319.

31. Fortina AB, Piaserico S, Caforio AL, Abeni D, Alaibac M, Angelini $A$, et al.Immunosuppressive level and other risk factors for basal cell carcinoma and squamous cell carcinoma in heart transplant recipients. Arch Dermatol. 2004;140:1079-85.

32. Jensen P, Hansen S, Moller B, Leivestad T, Pfeffer P, Geiran O, et al. Skin cancer in kidney and heart transplant recipients and different long-term immunosuppressive. J Am Acad Dermatol. 1999;40:177-86.

33. Salim A, Reece SM, Smith AG, Harrison D, Ramsay HM, Harden PN, et al. Sebaceous hyperplasia and skin cancer in patients undergoing renal transplant. J Am Acad Dermatol. 2006;55:878-81.

34. Mihalis EL, Wysong A, Boscardin WJ, Tang JY, Chren MM, Arron ST. Affecting sunscreen use and sun avoidance in a U.S. national sample of organ transplant recipients. Br J Dermatol. 2013;168:346-53.

35. Dal Sasso Mendes K, Murad F, Da Costa L, Prado K, Fontao M, Ohler L, et al. Photoeducation and photoprotection among liver transplant candidates. Gastroenterol Nurs. 2013;36:215-21.

36. Falk M, Magnusson H. Sun protection advice mediated by the general practitioner: An effective way to achieve long-term change of behaviour and attitudes related to sun exposure. Scand J Prim Heal Care. 2011;29:135-43. 
37. Bandi P, Cokkinides VE, Weinstock MA, Ward EM. Physician sun protection counseling: Prevalence, correlates, and association with sun protection practices among US adolescents and their parents, 2004. Prev Med. 2010;51:172-7.

38. Feuerstein I, Geller A. Skin cancer education in transplant recipients. Prog Transpl. 2008;18:232-41.

39. Rueda X, Acosta A, Aristizábal FE. Guías de práctica clínica para el tratamiento del carcinoma basocelular. Rev Asoc Colom Dermatol. 2008;16:102-17.
40. Acosta A, Rueda X, Alba PL. Guías de práctica clínica para el tratamiento del carcinoma escamocelular. Rev Asoc Colomb Dermatol. 2008;16:116-34.

41. Tejaswi M, Levender M, O`Neil J, West C, Pearce D, Feldman S. Incidence, risk factors, and preventive management of skin cancers in organ transplant recipients: A review of single and multicenter retrospective studies from 2006 to 2010. Dermatol Surg. 2013; 39:345-364

42. Liehn M, Fenske N, Glass F. Non-melanoma skin cancer in high-risk organ transplant recipients. Seminars in Oncology. 2012;39:134-8. 